\title{
Dynamic pH mapping in microfluidic devices by integrating adaptive coatings based on polyaniline with colorimetric imaging techniques $\uparrow$
}

\author{
Larisa Florea, Cormac Fay, Emer Lahiff, Thomas Phelan, Noel E. O'Connor, Brian Corcoran, Dermot \\ Diamond and Fernando Benito-Lopez*
}

\author{
${ }_{5}$ Received (in $\left.X X X, X X X\right)$ Xth $X X X X X X X X X 20 X X$, Accepted Xth XXXXXXXXX 20XX \\ DOI: $10.1039 / \mathbf{b 0 0 0 0 0 0 x}$
}

\begin{abstract}
In this paper we present a microfluidic device that has integrated $\mathrm{pH}$ optical sensing capabilities based on polyaniline. The optical properties of polyaniline coatings change in response to the $\mathrm{pH}$ of the solution that is flushed inside the microchannel offering the possibility of monitoring $\mathrm{pH}$ in continuous flow over a 10 wide $\mathrm{pH}$ range throughout the entire channel length. This work also features an innovative detection system for spatial localisation of chemical $\mathrm{pH}$ gradients along microfluidic channels through the use of a low cost optical device. Specifically, the use of a microfluidic channel coated with polyaniline is shown to respond colorimetrically to $\mathrm{pH}$ and that effect is detected by the detection system, even when $\mathrm{pH}$ gradients are induced within the channel. This study explores the capability of detecting this gradient by 15 means of imaging techniques and the mapping of the camera's response to its corresponding $\mathrm{pH}$ after a successful calibration process. The provision of an inherently responsive channel means that changes in the $\mathrm{pH}$ of a sample moving through the system can be detected dynamically using digital imaging along the entire channel length in real time, without the need to add reagents to the sample. This approach is generic and can be applied to other chemically responsive coatings immobilised on microchannels.
\end{abstract}

\section{${ }_{20}$ Introduction}

Conventional glass-type electrodes have been widely used for $\mathrm{pH}$ measures for many years in both industry and academic areas. However, in terms of specific applications (e.g. in vivo, food industry, or for clinical applications), they posses several ${ }_{25}$ disadvantages due to their size constraints, rigidity, and the inflexibility of the glass electrode. In recent years, a wide number of $\mathrm{pH}$ sensors have been developed to overcome these limitations, including ion sensitive field-effect transistor (iSFET) $\mathrm{pH}$ sensors ${ }^{1-4}$, optical $\mathrm{pH}$ sensors based on $\mathrm{pH}$ responsive dyes ${ }^{5-8}$, hydrogel 30 film $\mathrm{pH}$ sensors ${ }^{9,10}$, and solid-state metal oxides $\mathrm{pH}$ sensors ${ }^{11-13}$. In particular, optical $\mathrm{pH}$ sensors present several advantages over the traditional $\mathrm{pH}$ electrodes as such their low costs, immunity from electromagnetic field, absence of electric contacts, possibility of reference electrode removal and a high degree of ${ }_{35}$ miniaturisation ${ }^{14}$. Optical fiber-based $\mathrm{pH}$ sensors have been particularly popular, as the fibre allows the optical signal to be transported over long distances, which can facilitate applications in remote sensing ${ }^{15}$.

Usually, these optical pH sensors (or optrodes) employ a dye 40 or an indicator that requires immobilisation onto a solid support material. There are several critical issues related to this approach: firstly, the dye should retain its optical properties after the immobilisation process ${ }^{16}$ and secondly, it should not leach into the solution ${ }^{17}$. A third issue of practical importance is their 45 inherently narrow dynamic response range which is usually around 3-4 $\mathrm{pH}$-units centred on the dye's $\mathrm{pKa}^{18}$.

Therefore, the further improvement of such sensors focuses on the search for new materials that can overcome these issues. An alternative approach is to use the inherent optical response of 50 certain polymers like the conducting polymer polyaniline (PAni) rather than a conventional $\mathrm{pH}$-sensitive dye. PAni displays striking changes in the visible/NIR spectrum upon protonmediated doping-dedoping of the polymer backbone, thus offering the possibility of developing optical sensors with ${ }_{55}$ extended $\mathrm{pH}$ ranges. The polymer itself, therefore, acts as both the matrix support and the indicator dye. In this way, leaching is prevented, thereby enhancing the long-term stability and reproducibility.

PAni is an excellent candidate for the fabrication of optical ${ }_{60}$ sensors in the visible-near IR detection since it is an intrinsically $\mathrm{pH}$-sensitive polymer with good environmental stability ${ }^{19,20}$. Furthermore, by focusing on PAni nanofibres we can dramatically increase the surface area of the material ${ }^{21}$, which in turn can manifest in improved response times and sensitivity.

65 A relatively new and promising approach to produce sensors of this kind involves optofluidics wherein optical and fluidic functionalities are integrated at the micro- and nano-scale to leverage their combined advantages by functionalising the inner walls of a microchannel with a responsive material ${ }^{22,23}$. For 70 example, functionalisation with antibodies for flow-through cell separation have been reported ${ }^{24}$, as have florescent dyes for optical sensing of acidity ${ }^{25}$ and monolayers that exhibit metal ion sensing properties ${ }^{26,27}$.

Nowadays, a wide variety of detectors can be employed to 75 transduce the colorimetric analytical signal, such as light dependent resistors ${ }^{28,29}$, photodiodes $^{30-32}$, phototransistors ${ }^{33-35}$ and even reverse-biased LEDs ${ }^{36-38}$. More specific to this study is the use of digital imaging cameras for the detection of colorimetric reactions. Up to now, almost all of the studies carried out have 80 been based on the RGB colour model, probably because this is readily accessible via a number of popular image processing 
packages ${ }^{39-43}$. The major challenge with this approach is that the RGB colour space is inherently sensitive to changes in ambient lighting, and measurements therefore have to be made under strictly controlled light conditions. Recently, studies such as those ${ }_{5}$ by Fay et al. ${ }^{44}$ (qualitatively) and Cantrell et al. ${ }^{45}$ (quantitatively) explored the use of a different colour model (HSV) for colorimetric chemical analysis which has shown to be more tolerant of ambient light variations ${ }^{46}$.

Based on this concept, we present an innovative, robust, 10 simple and fast method to measure $\mathrm{pH}$ simultaneously at all locations across an entire microfluidic system using polyaniline nanofibres modified microchannel coupled with HSV-based digital image color analysis.

\section{Experimental}

\section{Microfluidic device fabrication}

The fabrication of the master mold was carried out using a laser ablation system-excimer/ $\mathrm{CO}_{2}$ laser (Optec Laser Micromachining Systems, Belgium) by cutting the microfluidic structures in a $50 \mu \mathrm{m}$ double-sided pressure sensitive adhesive

20 film, PSA, (AR8890, Adhesives Research, Ireland) and pasting one of the PSA sides to a petri dish ${ }^{47}$.

For PDMS casting, the precursor was prepared by mixing PDMS elastomer with the curing agent from Sylgard 184 kit at a weight ratio of 10:1, poured onto a master mould, and cured in an 25 oven at $80{ }^{\circ} \mathrm{C}$ for $2 \mathrm{~h}$. Following curing, the PDMS layer is peeled from the master. The inlets and outlets $(800 \mu \mathrm{m}$ in diameter) were made using a manual puncher (Technical Innovations, Inc., Brazoria, TX). The PDMS replica $(\sim 1 \mathrm{~mm}$ height) was thoroughly washed with isopropanol and exposed to

30 oxygen plasma to seal the chip to a clean glass slide (35 $\mathrm{mm}$ x 64 $\mathrm{mm}$, Agar Scientific Limited, England) or another flat PDMS layer $(\sim 2 \mathrm{~mm}$ height). Silicon tubes were employed to further connect the main inlets with a syringe pump (PHD 2000 Syringe, Harvard Apparatus) for sample delivery and washing.

\section{Microfluidic device functionalisation}

The functionalisation of the inner walls of the microfluidic channel with PAni nanofibres was achieved using the procedure described in Figure 1. The detailed microchannel

40 functionalisation process and the corresponding characterisation of the coating are listed in the ESI†. Briefly, immediately after exposure of the PDMS chips to oxygen plasma for $60 \mathrm{~s}$ (Harrick Plasma) and sealing to the glass slide/PDMS layer, the activated channels $(1000 \times 100 \mu \mathrm{m})$ were flushed with a $20 \%$ wt solution 45 of $\mathrm{N}$-[3- (trimethoxylsilyl) propyl]aniline in ethanol for $60 \mathrm{~min}$ at a flow rate of $0.5 \mu \mathrm{L} \mathrm{min}^{-1}$. Using this technique, a monolayer of silane-bearing aniline was formed on the substrate via molecular self-assembly. Chemical deposition of the PAni on the modified microchannel walls was performed by filling the microchannel

${ }_{50}$ with freshly prepared $1 \mathrm{M} \mathrm{HCl}$ solution containing the oxidant (ammonium peroxydisulfate) and aniline in a molar ratio of $0.25: 1$. This molar ratio was chosen as it has been previously shown that polymerisation of aniline in these experimental conditions produces nanofibres ${ }^{48}$. The pendant aniline on the 55 surface served as the initiation site for polymerisation and was also used to covalently anchor the PAni chain on the substrate ${ }^{49}$.
The polymerisation time was fixed to $20 \mathrm{~h}$. After polymerisation, the channels were washed extensively with water to remove any unattached polyaniline nanofibres. The resulting films had good 60 adhesion due to the chemical bonding between the substrate and polymer film. Homogeneous PAni coatings were obtained, covalently attached to the internal walls of microchannels made of PDMS/PDMS or PDMS/glass, see Figure 2.

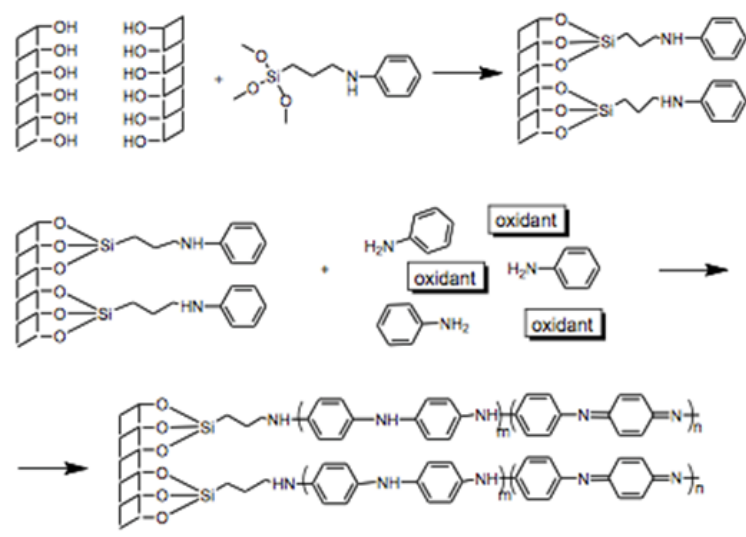

Figure 1. Chemical functionalisation of microchannel surface with polyaniline chains
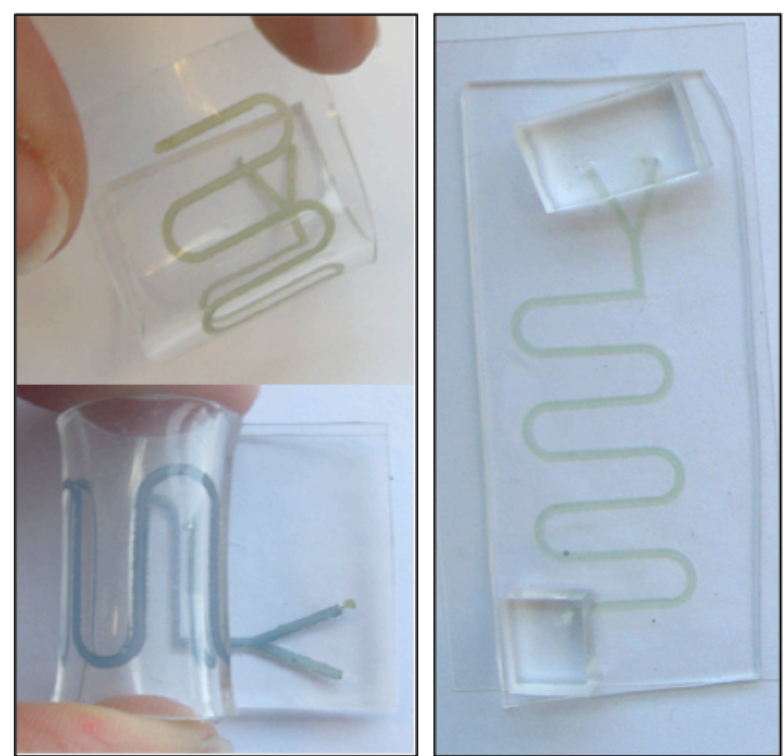

70 Figure 2. Pictures of polyaniline functionalised PDMS/PDMS (left) acidic on the top and basic on the bottom, and glass/PDMS (right) microfluidic devices. The picture on the right shows the PDMS extensions that were attached to the PDMS layer by oxygen plasma. These extensions were configured to secure the connections between the 75 silicon tubes and the inlets of the microchannel and to facilitate sample delivery.

\section{Measurement of absorbance spectra of PAni coatings}

Changes in the absorbance spectra of the PAni coatings as 80 different $\mathrm{pH}$ solutions flushed inside the microchannel were recorded using two fiber-optic light guides connected to a Miniature Fiber Optic Spectrometer (USB4000 - Ocean Optics) 
and aligned using an in-house made holder. The in-housedesigned holder was fabricated using a 3D printer (Dimension SST 768) in black acrylonitrile butadiene styrene co-polymer (ABS) plastic in order to minimise interferences from ambient ${ }_{5}$ light. The two parts of the holder ( 2 identical parts, one to be placed on top of the microfluidic chip, the other underneath) were designed using ProEngineer CAD/CAM software package and fixed together with screws ensuring precise alignment between the two fiber-optics (ESI - Fig. S1).

10 The Absorbance spectra recorded with Miniature Fiber Optic Spectrometer (USB4000). For clarity, all absorbance spectra recorded were smoothed using Origin software using SavitzkyGolay algorithm.

\section{Digital image capture}

After the fabrication of the flow channels and subsequent analysis using reference instrumentation (spectrophotometer) the channels were then subjected to analysis via digital imaging techniques. The channel was placed within the field of view of a standard 20 colour camera (Panasonic DMC-FZ38) along with a white background for subsequent image processing. An XRite professional colour reference chart was also placed within the camera's field of view (see ESI $\uparrow$ - Figure S2) as this experiment was performed under variable ambient lighting conditions.

25 Solutions of known $\mathrm{pH}$ produced by mixing appropriate amounts of hydrochloric acid or sodium hydroxide ( $\mathrm{pH} 2$ to 12) were then flushed through the microchannel at a flow rate of 50 $\mu \mathrm{L} \min ^{-1}$. At each $\mathrm{pH}$ unit step, an image was captured using the colour camera. This process was repeated and multiple images ${ }_{30}$ gathered at each unit step to investigate reproducibility.

An additional set of images were similarly captured, in which the flow channel was first filled with a solution of $\mathrm{pH} 3$ and then a droplet of $\mathrm{pH} 6.5$ aqueous solution placed at the opposite inlet where it was encouraged along the microchannel via an applied 35 negative pressure using a microsyringe. This generated a $\mathrm{pH}$ gradient inside the microchannel along the channel's length. Therefore, the channel showed two extreme colours at either end coupled with a colour gradient connecting them. The ability to detect this $\mathrm{pH}$ gradient point through image processing and 40 analysis was then investigated.

\section{Image processing}

A number of image processing steps were employed in order to analyse the overall channel $\mathrm{pH}$ for the calibration procedure, and ${ }_{45}$ subsequently additional steps were undertaken to detect the $\mathrm{pH}$ gradient along the channel. After capturing an image (see ESI $\uparrow$ Figure S2a) a segmentation process identified regions of interest from background areas (see ESI $\dagger$ - Figure S2b). The regions were identified based on their spatial coordinates and were matched 50 between progressive calibration images on this basis. The average Hue component of each region was taken to represent the colour of the channel and reference patches, which were then used for normalisation, and later to generate a calibration plot. Following this, a similar approach was employed to analyse the $\mathrm{pH}$ gradient 55 along the channel in which the $\mathrm{pH}$ analysis was localised at every point along the channel. A more detailed account of these processing steps can be found within the accompanying supporting information.

\section{${ }_{60}$ Results and discussion \\ Characterisation of the PAni coatings}

The PAni coatings were characterised by Raman spectroscopy as it permits in situ analysis ${ }^{50}$ of PAni coating inside the microchannel (see ESI $\dagger$ - Figure S4). Raman spectroscopy ${ }_{65}$ showed that PAni is obtained in its half-oxidised emeraldine state $^{51}$. In addition, Raman spectroscopy was also employed to study the changes in the bonding structure of the coatings upon doping-dedoping, as very distinct signature bands appear for the quinoid and benzenoid rings, respectively ${ }^{52,53}$. When a solution 70 of $\mathrm{pH} 2$ is passed through the microchannel, PAni presents the typical bands for the emeraldine salt (ES). When a solution of $\mathrm{pH}$ 12 is flushed through the microchannel, the ES bands decrease and the specific quinoid ring bands appear in the spectra, reflecting the dedoped state - emeraldine base (EB).

75 Scanning electron microscope (SEM) images showed that using the polymerisation technique described in the experimental section, PAni nanofibres were obtained, covalently attached to the inner walls of the microchannels, (Figure 3). The immediate advantages of having nanofibres (versus bulk PAni) are the high 80 surface area that is exposed to the target molecules and the very short diffusional path lengths ${ }^{54}$ which produces enhanced sensitivity coupled with fast response times ${ }^{21,55,56}$.

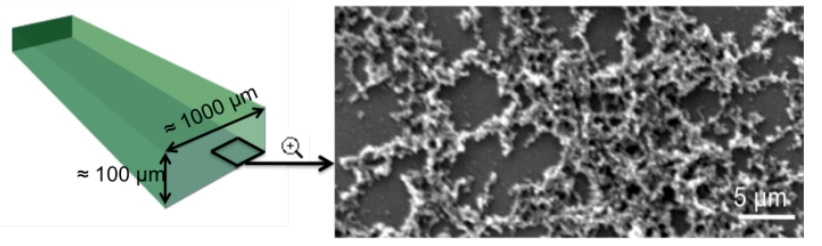

Figure 3 Representation of the polyaniline functionalised microchannel 85 (left). SEM image of the polyaniline functionalised glass bottom layer showing a homogeneously covered surface with polyaniline nanofibres (right).

\section{pH measurements}

Polyaniline's sensitivity towards $\mathrm{pH}$ has been extensively used in 90 recent years for the development of $\mathrm{pH}$ sensors due to its intrinsic

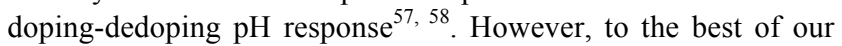
knowledge this is the first example of the use of polyaniline nanofibres as a $\mathrm{pH}$ optical sensor in a microfluidic device, wherein the whole inner wall of the microchannel acts as a sensor ${ }_{95}$ enabling the $\mathrm{pH}$ to be measured simultaneously at all points within the channel.

The reversible protonation/deprotonation reaction of PAni is of particular interest during the development of the $\mathrm{pH}$ sensor. The process occurs on the imine nitrogen atoms as shown in $\mathrm{ESI} \uparrow$ ${ }_{100}$ Figure S5. The transformation of Emeraldine Salt (ES) to Emeraldine Base (EB) by deprotonation is accompanied by significant changes in colour. This phenomenon is observed in the case of the PAni coatings when colourless solutions of at varying $\mathrm{pH}$ are passed through the microchannel, showing the 105 ability of the covalently bonded PAni nanofibres to rapidly respond to changes in their environment (see ESI $\uparrow$ - Figure S5). 
The changes in the absorbance spectra of the PAni coatings in response to different $\mathrm{pH}$ solutions flushed inside the microchannel were recorded using two fiber-optic light guides connected to a Miniature Fiber Optic Spectrometer and aligned 5 using an in-house made cell.

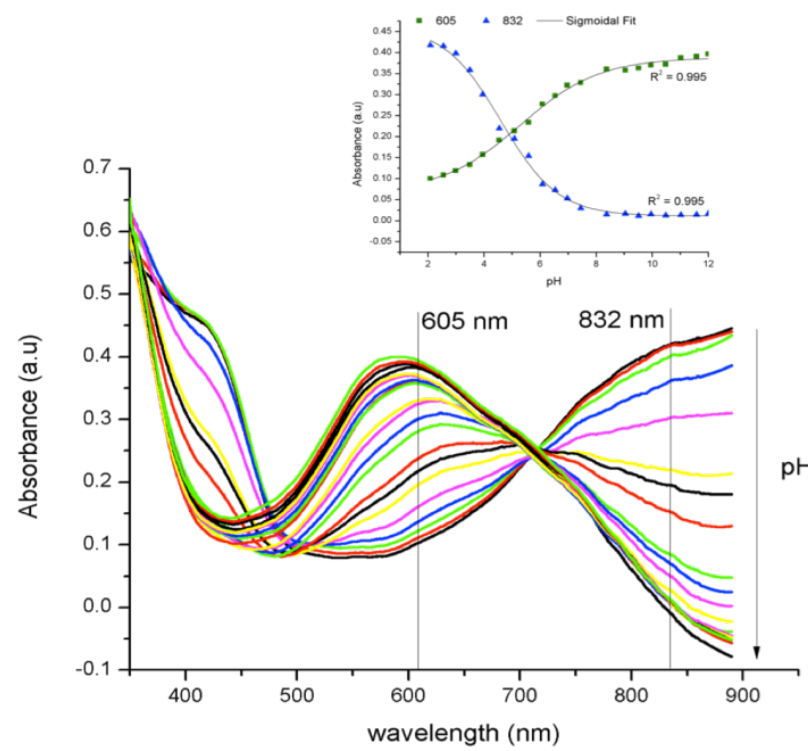

Figure 4. Absorbance spectra of the polyaniline coatings in the channel when solutions at different $\mathrm{pH}$ are passed through $(\mathrm{pH} 2-12)$. Inset Graph of the absorbance change of polyaniline coatings $v s$. $\mathrm{pH}$ at $605 \mathrm{~nm}$ 10 and $832 \mathrm{~nm}$.

The UV-VIS absorption spectra of PAni films were measured for each $\mathrm{pH}$ solution passed through the channel at a flow rate of 50 $\mu \mathrm{L} \mathrm{min}{ }^{-1}$, see Figure 4. It is important to note that the absorbance spectrum changes very rapidly after the solution reaches the 15 detection area (approx. $2 \mathrm{~s}$ ), thereby ensuring a very fast response of the device (see ESI $\uparrow$ - videos 1 and 2). Moreover, the signal remains stable during the timescale of the experiment, which was set to 5 minutes. The measurements were done in triplicates with a standard deviation of the absorbance value over the calibration 20 range of 0.001 .

As depicted in Figure 4 the spectrum of the PAni coatings is highly $\mathrm{pH}$ dependent and changes in colour from the green (ES) to blue (EB). Increasing the $\mathrm{pH}$ from 2 to 12 leads to a shift in the absorption $\lambda_{\max }$ of PAni in the visible region from $420 \mathrm{~nm}$ (at $\mathrm{pH}$ ${ }_{25}$ 2) to $605 \mathrm{~nm}$ (at pH 12). This shift is due to doping-dedoping of PAni coatings and can be explained by the different degree of protonation of the imine nitrogen atoms in the polymer chain ${ }^{59}$. More specifically, in a low $\mathrm{pH}$ environment, PAni exhibits strong absorbances at approximately $420 \mathrm{~nm}$ and $830 \mathrm{~nm}$ assigned to 30 polaron and bipolaron transitions. Upon dedoping these transitions disappear and a new absorbance band appears at approximately $600 \mathrm{~nm}$. This new band is ascribed to the exciton formation in the quinonoid rings ${ }^{60}$ and it is responsible for the blue colour of the PAni coatings. The $\mathrm{pH}$ dependence of the 35 absorptions at $605 \mathrm{~nm}$ and $832 \mathrm{~nm}$ were plotted in Figure $4-$ Inset. The characteristic PAni sigmoid shape curve $\left(\mathrm{R}^{2}=0.996\right)$ was obtained for the absorbance change versus $\mathrm{pH}$, similar to the previous reported results in the case of PAni ${ }^{19}, 51$. The curve is broad, ensuring that the $\mathrm{pH}$ dependence of the PAni occurs over a 40 wide range of $\mathrm{pH}$. Therefore, this type of coating can be used for
$\mathrm{pH}$ sensing across a reasonably broad range, constituting an important advantage over common $\mathrm{pH}$ indicator dyes. Since PAni does not fit the expected response curve for an indicator, because of its broad range, the Henderson-Hasselbach equation cannot be ${ }_{45}$ applied $^{7}$. In the literature, there have been attempts to introduce an adjusted exponent to fit PAni doping- dedoping behaviour using Henderson-Hasselbach equation, although these attempts were proven to be unsuccessful ${ }^{58}$. Therefore, only an apparent $p K a$ value can be used to describe the sensor response. As ${ }_{50}$ depicted from Figure 4 - Inset, the apparent $p K a$ value of PAni coatings is approximately 5 , representing the $\mathrm{pH}$ value where the two sigmoidals intersect (concentration of ES is approximately equal with the concentration of EB). Most probably this value represents a distribution of $p K a^{\prime} s$ of PAni units with differing

${ }_{55}$ chain length and local environments ${ }^{57}$. Nevertheless, since important changes in $\lambda_{\max }$ and absorbance were observed in the $\mathrm{pH}$ range from 2 to 8 , it should be possible to use these PAni coated microchannels for monitoring the $\mathrm{pH}$ of, for instance, physiological fluids (gastric juice, saliva, blood) which are 60 important applications for microfluidics at this time.

\section{pH determination via colorimetric imaging analysis}

It has been shown that the channels respond accurately to changes in $\mathrm{pH}$ when analysed using a spectrophotometer. However, this ${ }_{65}$ work also explored the possibility of performing colorimetric analysis without the need for specialised instrumentation i.e. through the use of a standard digital colour camera. This has the potential to extend the applicability of the sensor, for instance, to point of care microfluidic devices and to low cost diagnostics for 70 the developing world using mobile phones with integrated digital cameras to capture analytical information ${ }^{61}$. The steps taken to extract colorimetric information from the captured images are outlined in detail in the ESI+.

Processing of the images began with the application of a white 75 balance algorithm. This was possible as the image setting/scene was relatively similar in each case, and included a white region specifically for this purpose. As this study took place in an ambient lighting environment, normalisation of the images in this way was necessary to compensate for shifts in ambient light 80 intensity. For further robustness, the Hue value representing the colour of the channel underwent a colour normalisation process using two of the array of invariant reference patches (i.e. the purple and yellow patches as presented in Figure S2). Next, the average and standard deviation of the channel's normalised Hue 85 value across each of the captured images at corresponding $\mathrm{pH}$ unit steps were calculated (RSD $\leq 2.06 \%$ ). Following this, a calibration plot of the camera's response to different $\mathrm{pH}$ solutions was achieved and a sigmoidal model was applied to the data points, see ESI $\dagger$ - Figure S6. It can be seen from the figure that an 90 excellent fit was achieved $\left(\mathrm{R}^{2}=0.998, \mathrm{n}=18\right)$ and subsequently, the resulting mathematical model was used to map the camera response to $\mathrm{pH}$ concentration values for gradient analysis.

While spectrophotometer and the camera generate the $\mathrm{pH}$ estimations on the basis of 'colour' measurements, the way in 95 which this is achieved is different for both devices. The spectrophotometer can generate a calibration plot at any effective wavelength (i.e. regions of the absorbance spectrum of the dye that change with $\mathrm{pH}$ ) within its measurement range. For this 
study, the most dominant peak changes were selected; $605 \mathrm{~nm}$ and $832 \mathrm{~nm}$, see Figure 4. In contrast, the camera measures colour across the entire available spectrum, and wavelength specific measurements are not possible except through the rather 5 crude RGB division of the spectrum into three 'Red', Green' and 'Blue' (RGB) channels. When conversion into the HSV colour space is applied, the $\mathrm{H}$ (Hue) component quantitatively represents the most dominant 'colour' based on the transformation from the captured RGB data to the target HSV colour space ${ }^{46}$, see Figure ${ }_{10} \mathrm{~S} 6$.

Although, individually, each approach generated good quality fits to the calibration data, it was important to establish whether a correlation existed between both detection methods. One way of achieving this was to compare the predicted $\mathrm{pH}$ at each unit using 15 the derived mathematical regression models. Figure S7 presents a plot of the predicted $\mathrm{pH}$ using the camera model against the UVvis model at $832 \mathrm{~nm}$. Clearly, a linear correlation exists when comparing both approaches with a good fit resulting $\left(\mathrm{R}^{2}=0.98, \mathrm{n}\right.$ $=18$ ). Moreover, the difference in slope between this linear fit 20 and the ideal slope (slope $=1$ ) is relatively small at 0.021 , suggesting there is little bias or skewness between the two data sets. The correlation between the camera and the UV-Vis model at $605 \mathrm{~nm}$ was also investigated. Similar accuracies were achieved with $\mathrm{R}^{2}=0.98$ and a difference to the ideal slope of ${ }_{25}$ 0.025. From Figure S7 it can be seen that the goodness of fit of the correlation decreases above c.a. $\mathrm{pH}$ 7. This arises from the increasingly small absorbance (colour changes) occurring above this value, see Figure 4 (inset). Despite this, it is interesting to note that the camera and the spectrometer data remain reasonably 30 well correlated above $\mathrm{pH} \mathrm{7,} \mathrm{although} \mathrm{the} \mathrm{scatter} \mathrm{is} \mathrm{understandably}$ greater, see ESI $\uparrow$ - Figure S7.

\section{Gradient pH measurements}

The main advantage of the digital camera over the 35 spectrometer lies in its ability to rapidly generate spatially distributed information. This should enable the camera to dynamically track changes in $\mathrm{pH}$ along the entire length of the fluidic channel. To test this thesis, the microchannel was filled with a $\mathrm{pH} 3$ solution and a second solution of $\mathrm{pH} 6.5$ was 40 introduced at one end, as described in the experimental section and ESI $\dagger$. The solutions were allowed to diffuse until a $\mathrm{pH}$ gradient visually appeared whereupon an image was captured (Figure 5).

Following the image processing and data extraction as ${ }_{45}$ described above, the images were white balanced and the Hue values at each localised point normalised with respect to the colour reference patches, as described previously for the calibration process. Using the calibration model derived from the sigmoidal regression analysis, the localised Hue values were 50 mapped to their corresponding $\mathrm{pH}$ concentrations and a plot of $\mathrm{pH}$ concentration over the length of the channel was derived. To reduce noise, a smoothing algorithm based on the Savitzky-Golay filter ${ }^{62}$ was applied to the data set. For both Figure $5 \mathrm{~b}$ and Figure $5 \mathrm{c}$, labels are present to denote discrete points along the flow path 55 i.e. points of maximum curvature along the four considered flow bends.

It can be seen visually from Figure $5 \mathrm{~b}$ that the $\mathrm{pH}$ varies significantly between points ' 2 ' and ' 3 ' as reflected in the colour gradient. Correspondingly, the analysis presented (Figure 5c) 60 shows a dramatic change in $\mathrm{pH}$ from $c a$. $\mathrm{pH} 3$ at ' 2 ' to $c a$. $\mathrm{pH} 6.5$ at, and beyond, point ' 3 '. Figure $5 \mathrm{c}$ shows the resulting $\mathrm{pH}$ gradient as estimated by processing the colour image with the digital camera algorithm. Close examination of the image reveals the presence of small bubbles, which manifest as slight ${ }_{65}$ inconsistencies in the unsmoothed $\mathrm{pH}$ data in Figure 5c.

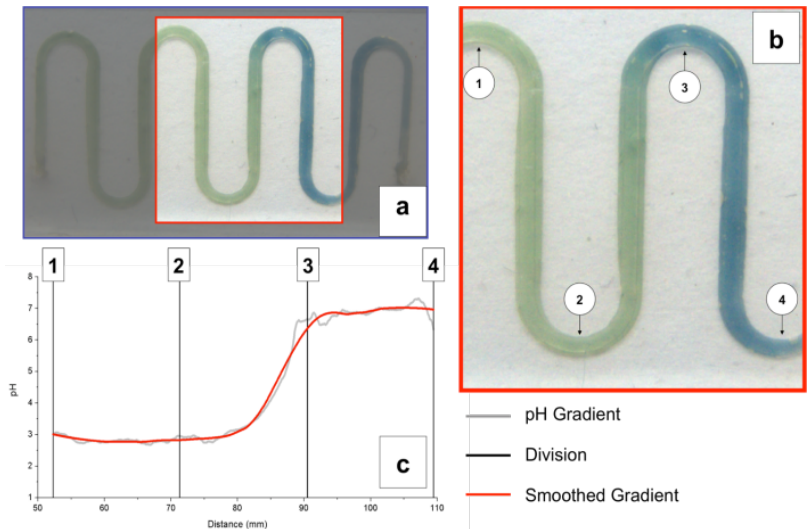

Figure 5. (a) Images showing a $\mathrm{pH}$ gradient along the microfluidic channel reflected in the changing colour of the PAni coating, red box shows a magnified section in (b), which also identifies specific locations 70 (1-4) highlighted in (c), Plot of the $\mathrm{pH}$ gradient along the flow channel generated from the image. The grey line is the raw data from the analysis; the red line has been smoothed using the Savitsky-Golay algorithm.

To demonstrate the capabilities of the digital imaging approach, changes in the $\mathrm{pH}$ along the entire channel were 75 tracked using low-cost digital video imaging, see supplementary information (ESI $\uparrow-$ Video 3). In this example, the image section under analysis is bounded by a red square and also enlarged (shown in the top left corner). In addition, a dynamic plot is presented on the right hand side of the video showing the change ${ }_{80}$ in $\mathrm{pH}$ along the channel. Although the data in Figure 5a and ESI $\dagger$ - Video 3 represents the $\mathrm{pH}$ dynamics of the channel at a single point in time, this process can be easily expanded to enable $\mathrm{pH}$ variations to be tracked dynamically along the entire microfluidic system as a function of time.

85 This capability could have a substantial impact in many areas. For instance, a number of chemical reactions are time critical and require precision when introducing a reagent. By coupling a microfluidic system, PAni and a low cost colour camera, a miniaturised and cost effective solution can be achieved for many 90 chemical and biochemical sensing scenarios that rely on a localised $\mathrm{pH}$ to drive the reaction. The speciation state of multibasic acids, or of amino acids could be inferred from a knowledge of $\mathrm{pH}$ gradients. Mixing processes involving buffers could be tracked to identify locations of optimum $\mathrm{pH}$ for particular ${ }_{95}$ processes, and to explore how these locations can be moved, expanded or contracted prior to addition of active reagents. Furthermore, the rapid improvement in price-performance in digital photography through the development of sophisticated, miniaturised and low cost CCD sensors ${ }^{63-65}$ and its increasing 100 integration with mobile phones, provides a powerful technology platform for many new applications. However, while tremendous potential impact of integration of chemical/biological measurements with digital imaging and communications is 
compelling, for example, in tele-health and personal (point-ofneed) diagnostics, the route to winning applications is not clear. Hence, companies like Nokia are sponsoring global competitions with very significant prizes to generate ideas from which they 5 will select the best possible candidate applications ${ }^{66}$. This activity emphasises the rising importance of applying digital imaging to analytical measurements.

\section{Conclusions}

A new, simple, and fast photometric method to measure $\mathrm{pH}$ using 10 PAni based coatings in microfluidic devices is presented. $\mathrm{pH}$ measurements were performed in continuous flow mode using fiber-optic light guides aligned to the device using an in-house made cell. The main advantage of these sensors is that no reagent indicator is needed to measure the $\mathrm{pH}$, because the PAni acts as

15 the indicator itself, reducing the complexity of $\mathrm{pH}$ detection inside microchannels. The functionalisation process is easy and highly reproducible from microdevice to microdevice and over the whole microchannel length. Moreover, it can be easily achieved using different materials as such glass, PDMS or any 20 other material that allows the introduction of hydroxyl groups on the surface, necessary for the present silanisation procedure. Although the time for functionalisation is rather long $(\sim 22 \mathrm{~h})$, the polyaniline functionalised micro-channels are suitable for multiple uses since the coating can be easily regenerated by

25 passing an acidic solution ( $\mathrm{HCl}$ solution, $\mathrm{pH} 2$ ) inside the channel. PAni coated microchannels present long-term stability and reproducibility, can be stored at room temperature, exposed to air, empty or filled with a $\mathrm{pH} 2$ solution for over two months without any deterioration in sensor performance.

30 Since this technique is based on $\mathrm{pH}$ responsive coatings, at present, it is not suitable for $3 \mathrm{D} \mathrm{pH}$ sensing in microchannels but rather 2D (along the length and width of the channel) $\mathrm{pH}$ sensing. However, when coupled with imaging techniques, this approach offers a low cost, accurate approach for the tracking of the $2 \mathrm{D}$

35 temporal and spatial dynamics of $\mathrm{pH}$ changes along an entire microfluidic channel in real-time, without the need to add a $\mathrm{pH}$ sensitive dye to the liquid phase in the channel. The approach of using the colour camera for $\mathrm{pH}$ mapping is generic and could be applied for a wide variety of $\mathrm{pH}$ responsive systems.

\section{${ }_{40}$ Acknowledgements}

The project has been carried out with the support of the Irish Research Council (IRC) - Embark Initiative and Science Foundation Ireland under the CLARITY initiative, grant 07/CE/I1147.

\section{${ }_{45}$ Notes and references}

CLARITY: Centre for Sensor Web Technologies, National Centre for Sensor Research, Dublin City University, Dublin, Ireland, Fax: +3531 700 7995,Tel:+3531700 7699;*E-mail:fernando.lopez@dcu.ie ;

$\uparrow$ Electronic Supplementary Information (ESI) available: Video 1 and

${ }_{50}$ Video 2 are showing the rapid colour change of the polyanile coating when solutions of different $\mathrm{pHs}$ are passed through the microchannel. In Video 3, using image processing techniques, $\mathrm{pH}$ is succesfully mapped when a $\mathrm{pH}$ gradient is induced inside the polyaniline modified microchannel. See DOI: 10.1039/b000000x/
1. K. Pasztor, A. Sekiguchi, N. Shimo, N. Kitamura and H. Masuhara, Sens. Actuators, B, 1993, 12, 225-230.

2. V. K. Khanna, Indian Journal of Pure \& Applied Physics, 2012, 50, 199-207.

60 3. B. Nemeth, S. Tsuda, C. Busche, L. Cronin and D. R. S. Cumming, Electron. Lett, 2012, 48, 143-U120.

4. Y. L. Chin, J. C. Chou, T. P. Sun, W. Y. Chung and S. K. Hsiung, Sens. Actuators, B, 2001, 76, 582-593.

5. Y. Cheng, X. Luo, J. Betz, S. Buckhout-White, O. Bekdash, G. F. Payne, W. E. Bentley and G. W. Rubloff, Soft Matter, 2010, 6, 3177-3183

6. T. Gunnlaugsson, P. E. Kruger, P. Jensen, J. Tierney, H. D. P. Ali and G. M. Hussey, J. Org. Chem., 2005, 70, 10875-10878.

7. N. Klauke, P. Monaghan, G. Sinclair, M. Padgett and J. Cooper, Lab $70 \quad$ Chip, 2006, 6, 788-793.

8. X. Ge, Y. Kostov, L. Tolosa and G. Rao, Anal. Chim. Acta, 2012, 734, 79-87.

9. A. Richter, G. Paschew, S. Klatt, J. Lienig, K.-F. Arndt and H.-J. P. Adler, Sensors, 2008, 8, 561-581.

75 10. Q. T. Trinh, G. Gerlach, J. Sorber and K.-F. Arndt, Sens. Actuators, $B, 2006,117,17-26$.

11. Y. Cheng, P. Xiong, C. S. Yun, G. F. Strouse, J. P. Zheng, R. S. Yang and Z. L. Wang, Nano Lett., 2008, 8, 4179-4184.

12. C. N. Tsai, J. C. Chou, T. P. Sun and S. K. Hsiung, Sens. Actuators, 80 $B, 2005, \mathbf{1 0 8}, 877-882$.

13. W.-D. Huang, H. Cao, S. Deb, M. Chiao and J. C. Chiao, Sensors and Actuators a-Physical, 2011, 169, 1-11.

14. S. Martellucci, A.N. Chester and A. G. Mignani, Springer, New York, USA, 2000

85 15. R. M. Wolthuis, D. Saaski, E. Hartl, J. Mitchell, G., IEEE Trans. Biomed. Eng., 1992, 39, 531 - 537.

16. F. Buchholz, N. Buschmann and K. Cammann, Sens. Actuators, B, 1992, 9, 41-47.

17. B. Kuswandi and R. Narayanaswamy, Fresenius J. Anal. Chem., 1999, 364, 605-607.

18. R. W. Sabnis;, S. Sanders; and L. Dempsey, Handbook of Acid-Base Indicators, CRC Press, 2007.

19. Z. F. Ge, C. W. Brown, L. F. Sun and S. C. Yang, Anal. Chem., 1993, 65, 2335-2338.

95 20. E. K. Asijati, B.; Arifah, N.F.; Kurniawati, Y.I.; Gani, A.A.; Sensors and the International Conference on new Techniques in Pharmaceutical and Biomedical Research, 2005 Asian Conference on 2005, 111 - 114

21. J. X. Huang, S. Virji, B. H. Weiller and R. B. Kaner, J. Am. Chem. $100 \quad$ Soc., 2003, 125, 314-315.

22. L. Florea, A. Hennart, D. Diamond and F. Benito-Lopez, Sens. Actuators B: Chem., 2012, 175, 92-99.

23. L. Florea, F. Benito-Lopez, A. Hennart and D. Diamond, Procedia Engineering, 2011, 25, 1545 - 1548.

105 24. J. Miwa, Suzuki, Y., Kasagi, N., J. Microelectromech. Syst., 2008, 17, 611-622.

25. P. Mela, S. Onclin, M. H. Goedbloed, S. Levi, M. F. Garcia-Parajo, N. F. van Hulst, B. J. Ravoo, D. N. Reinhoudt and A. van den Berg, Lab Chip, 2005, 5, 163-170. 
26. L. Basabe-Desmonts, F. Benito-Lopez, H. J. G. E. Gardeniers, R Duwel, A. van den Berg, D. N. Reinhoudt and M. CregoCalama, Anal. Bioanal.Chem., 2008, 390, 307-315.

27. F. Benito-Lopez, S. Scarmagnani, Z. Walsh, B. Paull, M. Macka and D. Diamond, Sens. Actuators, B, 2009, 140, 295-303.

28. K. T. Lau, R. Shepherd, D. Diamond and D. Diamond, Sensors, 2006, 6, 848-859.

29. F. A. A. Matias, M. Vila and M. Tubino, Sens. Actuators, B, 2003, 88, 60-66.

10 30. G. J. Schmidt and R. P. W. Scott, Analyst, 1984, 109, 997-1002.

31. J. R. Clinch, P. J. Worsfold and H. Casey, Anal. Chim. Acta, 1987, 200, 523-531.

32. P. C. Hauser, S. S. Tan, T. J. Cardwell, R. W. Cattrall and I. C. Hamilton, Analyst, 1988, 113, 1551-1555.

15 33. K. S. Johnson, C. L. Beehler and C. M. Sakamotoarnold, Anal. Chim. Acta, 1986, 179, 245-257.

34. D. Betteridge, W. C. Cheng, E. L. Dagless, P. David, T. B. Goad, D. R. Deans, D. A. Newton and T. B. Pierce, Analyst, 1983, 108, 1-16.

20 35. M. A. Feres and B. F. Reis, Talanta, 2005, 68, 422-428.

36. K. T. Lau, S. Baldwin, R. L. Shepherd, P. H. Dietz, W. S. Yerzunis and D. Diamond, Talanta, 2004, 63, 167-173.

37. K. T. Lau, S. Baldwin, M. O'Toole, R. Shepherd, W. J. Yerazunis, S. Izuo, S. Ueyama and D. Diamond, Anal. Chim. Acta, 2006, $\mathbf{5 5 7 , 1 1 1 - 1 1 6 . ~}$

38. M. O'Toole, K.-T. Lau, B. Shazmann, R. Shepherd, P. N. Nesterenko, B. Paull and D. Diamond, Analyst, 2006, 131, 938-943.

39. S. M. Barnard and D. R. Walt, Nature, 1991, 353, 338-340.

40. I. Alexandre, S. Hamels, S. Dufour, J. Collet, N. Zammatteo, F. De Longueville, J. L. Gala and J. Remacle, Anal. Biochem., 2001, 295, 1-8.

41. L. Byrne, J. Barker, G. Pennarun-Thomas, D. Diamond and S. Edwards, TrAC, Trends Anal. Chem., 2000, 19, 517-522.

42. L. Byrne, K. T. Lau and D. Diamond, Irish Journal of Agricultural 35 and Food Research, 2003, 42, 119-129.

43. A. Lapresta-Fernandez and L. F. Capitan-Vallvey, Anal. Chim. Acta, 2011, 706, 328-337.

44. C. Fay, K.-T. Lau, S. Beirne, C. O. Conaire, K. McGuinness, B. Corcoran, N. E. O'Connor, D. Diamond, S. McGovern, G. Coleman, R. Shepherd, G. Alici, G. Spinks and G. Wallace, Sens. Actuators, B, 2010, 150, 425-435.

45. K. Cantrell, M. M. Erenas, I. de Orbe-Paya and L. F. CapitanVallvey, Anal. Chem., 2010, 82, 531-542.

46. A. R. Smith, SIGGRAPH Comput. Graph., 1978, 12, 12-19.

45 47. A. B. Shrirao and R. Perez-Castillejos, Chips \& Tips, 17 May 2010.

48. J. F. Qiang, Z. H. Yu, H. C. Wu and D. Q. Yun, Synth. Met., 2008, 158, 544-547.

49. C. G. Wu and J. Y. Chen, Chem. Mater., 1997, 9, 399-\&.

50. J. Dambrine, B. Geraud and J. B. Salmon, New J. Phys., 2009, 11.

50 51. K. Berrada, S. Quillard, G. Louarn and S. Lefrant, Synth. Met., 1995, 69, 201-204.

52. M. Bartonek, N. S. Sariciftci and H. Kuzmany, Synth. Met., 1990, 36, 83-93.

53. A. Hugotlegoff and M. C. Bernard, Synth. Met., 1993, 60, 115-131.

55 54. J. X. Huang, Pure and Applied Chemistry, 2006, 78, 15-27.
55. J. Huang, S. Virji, B. H. Weiller and R. B. Kaner, Chemistry-a European Journal, 2004, 10, 1315-1319.

56. S. Virji, J. D. Fowler, C. O. Baker, J. X. Huang, R. B. Kaner and B. H. Weiller, Small, 2005, 1, 624-627.

60 57. E. Pringsheim, E. Terpetschnig and O. S. Wolfbeis, Anal. Chim. Acta, 1997, 357, 247-252.

58. U. W. Grummt, A. Pron, M. Zagorska and S. Lefrant, Anal. Chim. Acta, 1997, 357, 253-259.

59. J. C. Chiang and A. G. Macdiarmid, Synth. Met., 1986, 13, 193-205.

65 60. A. J. Epstein, J. M. Ginder, F. Zuo, R. W. Bigelow, H. S. Woo, D. B. Tanner, A. F. Richter, W. S. Huang and A. G. Macdiarmid, Synth. Met., 1987, 18, 303-309.

61. A. W. Martinez, S. T. Phillips, G. M. Whitesides and E. Carrilho, Anal. Chem., 2010, 82, 3-10.

70 62. A. Savitzky and M. J. E. Golay, Anal. Chem., 1964, 36, 1627-\&.

63. H. Tamayama, K. Ito and T. Nishimura, Technology trends of highdefinition digital still camera systems, 2002.

64. P. Maxwell, Test for low cost CMOS image sensors, 2005.

65. F. Xiao, X. Zhang and B. Fowler, Color processing in camera

66. http://www.nokiasensingxchallenge.org/. 